\title{
The Theory of Von Klitzing's Constant and Phases
}

\author{
Keshav N. Shrivastava, Fellow, IACSIT
}

\begin{abstract}
The Hall resistivity is found to become a function of spin. For positive spin, one value is found but for negative sign in the spin, another value occurs. In this way, there is never only one value of the resistivity but there is doubling of values. The value of the von Klitzing's constant is a special case of more general dependence of resistivity on the spin. We investigate the effect of Landau levels. For extreme quantum limit, $n=0$, the effective charge of the electron becomes (1/2) $\mathrm{e}$. The fractional charge arises for finite value of the angular momentum. There is a formation of spin clusters. As the field increases, there is a phase transition from spin $1 / 2$ to spin $3 / 2$ so that $g$ value becomes 4 and various values of $n$ in Landau levels, $g(n+1 / 2)$, form plateaus in the Hall resistivity. For finite values of the orbital angular momenta, many fractional charges emerge. The fractional as well as the integral values of the charge are in full agreement with the experimental data. The generalized constant is $h /[(1 / 2)$ ge $]$ e which under special conditions becomes $\mathrm{h} / \mathrm{e}^{2}$ which is the von Klitzing's constant.
\end{abstract}

Index Terms-Von Klitzing's constant, g values, charge square and Planck's contant ratio, angular momentum.

\section{INTRODUCTION}

The resistivity at the plateaus is quantized in the units of $h / e^{2}$. Usually, the electron is associated with the electromagnetic field, the same way as the charge density is, in the Maxwell equations. The electric and magnetic field vectors are linked to the charge density. However, the charge is defined in such a way that the effect of self electromagnetic fields is already included in the value of the charge,

$$
e=1.602176487(40) \times 10^{-19} \text { Coulomb }
$$

The Planck's constant is associated with the frequency or the wave length of a particle,

$$
h=6.62606896(33) \times 10^{-34} \mathrm{Js} .
$$

It is a matter of a pencil calculation to show that,

$$
h / e^{2}=25812.8075651 \mathrm{Ohm} .
$$

This is called "one von Klitzing" constant because it was first measured by von Klitzing, Dorda and Pepper [1]. In their paper, the value given is $25813 \Omega$. The calculation of $\mathrm{h} / \mathrm{e}^{2}$ does not require that there should be two dimensionality or there should be Landau levels. However, the experimental value requires the Hall geometry. The value of $\mathrm{h} / \mathrm{e}^{2}$, does not require any electrodynamic correction. The fine structure

Manuscript received June 30, 2013; revised September 4, 2013.

Keshav N. Shrivastava is with the University of Hyderabad, Hyderabad 500046, India (e-mail: keshav1001@yahoo.com). constant is defined in such a way that,

$$
h / e^{2}=\mu_{\mathrm{o}} c /(2 \alpha)
$$

where $\mu_{0}=4 \pi \times 10^{-7} \mathrm{H} / \mathrm{m}$ and $\mathrm{c}$ is the velocity of light. The above expression is

$$
\alpha=\frac{e^{2}}{4 \pi \varepsilon_{o} \hbar c}
$$

where $\varepsilon_{0}=1 /\left(\mu_{0} c^{2}\right)$. At the present time, the value of the inverse fine structure constant [2] is, $1 / \alpha=137.035999$ 084(51) which is another way of writing the value of $\mathrm{h} / \mathrm{e}^{2}$. These are one and the same and not two different quantities. How the accuracy has become so high is another question but in 1965 , the value was $137.0388(6)$. The gyromagnetic ratio of the electron is given by [2],

$$
g / 2=1.00115965218073(28)
$$

This value is related to the fine structure constant,

$$
g / 2=1+C_{2}(\alpha / \pi)
$$

In this way, $g$ is related to $\alpha$ and $\alpha$ determines $h / e^{2}$. However, $g$ is subject to the electrodynamical corrections whereas $\mathrm{h} / \mathrm{e}^{2}$ is not. The electron is associated with the electromagnetic field because of the charge. The electromagnetic field is quantized in terms of photons. Therefore, there are many Feynmann diagrams which describe the electron-photon interaction so that many more terms arise in (7) which have to be carefully added. The Lande's formula gives,

$$
g / 2=1
$$

for $l=0$ and the electrodynamic correction is,

$$
\left.\frac{g}{2}\right|_{\text {electrodynamic }}=0.00115965218073(28)
$$

so that,

$$
\frac{g}{2}=\left.\frac{g}{2}\right|_{\text {Lande }}+\left.\frac{g}{2}\right|_{\text {electrodynamic }} .
$$

The $g$ value can be separated into electrodynamic part and Lande's part but in the case of the value of the charge such a separation is not available. The Lande's formula does not contain the electrodynamics but it contains the angular momenta, L, S and J. If there is any correction to the value of the charge due to the electrodynamics, it is already included in the tabulated value of e. There is a problem of gauge 
invariance according to which h/e is fixed and only one e in $\mathrm{h} / \mathrm{e}^{2}$ is subject to measurement. If both values of e are equal we get the $\mathrm{h} / \mathrm{e}^{2}$. In our theory [3]-[8] the resistivity is,

$$
\rho=\frac{h}{\frac{1}{2} g e^{2}}
$$

where $(1 / 2) g$ does not include the electrodynamic correction. In fact, such electrodynamic corrections are already included in $h / e^{2}$. We use the definition $\mathrm{g}=(2 j+1) /(2 l+1)$ so that for $j=l$ $\pm s$, there are two values of $\mathrm{g}$ which we call $\mathrm{g}_{ \pm}$,

$$
g_{ \pm}=\frac{2(l \pm s)+1}{2 l+1}
$$

Note that this value of $g_{ \pm}$, does not have the electrodynamic correction. The expressions (4), (5) and (7) suggest that $\mathrm{h} / \mathrm{e}^{2}$ is equivalent to $\alpha$ and (7) relates $\alpha$ to $g$ value. When $l=0$,

$$
g_{ \pm}=2( \pm s)+1
$$

For $s=1 / 2$ for + sign, $g_{+}=2$ so that $(1 / 2) g_{+}=1$ and the result (11) gives $h / e^{2}$. For $s=1 / 2$ and negative sign, $g_{-}=0$ and we get $\rho \rightarrow \infty$, or the conductivity, $\sigma \rightarrow 0$. We call these values as von Klitzing constants, which now have two values,

$$
R_{K}=h / e^{2}
$$

and

$$
R_{K}=\infty
$$

For $l=1, s=1 / 2$ for positive sign, (12) gives,

$$
g_{+}=\frac{2\left(1+\frac{1}{2}\right)+1}{3}=\frac{4}{3}
$$

or $(1 / 2) g_{+}=2 / 3$, which makes von Klitzing value,

$$
R_{K}=\frac{h}{\frac{2}{3} e^{2}}
$$

For $l=1, s=1 / 2$ and negative sign in (12),

$$
\mathrm{g}=\frac{2\left(1-\frac{1}{2}\right)+1}{3}=\frac{2}{3}
$$

or $(1 / 2) g_{-}=1 / 3$ so that the von Klitzing resistivity becomes,

$$
R_{K}=\frac{h}{\frac{1}{3} e^{2}}
$$

In this way many values of the Klitzing constant can be predicted. The fractional values calculated here agree with the measured values of Tsui, Stormer and Gossard [9] as well as grapheme [10].

\section{THE THEORY}

The eigen values of the harmonic oscillator are given by,

$$
E_{n}=\left(n+\frac{1}{2}\right) \hbar \omega
$$

where,

$$
\hbar \omega=g \frac{e \hbar}{2 m c} B .
$$

For $n=0, E_{0}=(1 / 2) \hbar \omega$ so that the frequency becomes,

$$
E_{0}=\frac{1}{2} \hbar \omega=\frac{1}{2} g \frac{e \hbar}{m c} B
$$

This means that we can replace e by $(1 / 2)$ ge or $e^{*}=(1 / 2) g e$. The von Klitzing resistivity now becomes,

$$
R_{K}=\frac{h}{\frac{1}{2} g_{ \pm} e^{2}}
$$

where we can generate a lot of values by changing $l$ and s but it is clear that there are pairwise values, due to \pm and not single value. There is a doubling of values. From (12) we can calculate the values of $g_{ \pm}$for various values of $l$ and $\mathrm{s}$ which gives values of the resistivity. We use the harmonic oscillator type expression, so that (23) becomes,

$$
R_{K}=\frac{h}{\left(n+\frac{1}{2}\right) g_{ \pm} e^{2}} .
$$

For, $n=0,1,2,3,4,5,6$, the values of $n+(1 / 2)$ are, $0,3 / 2$, $5 / 2,7 / 2,9 / 2, \ldots$

For $S=3 / 2, l=0$ we have for the positive sign, $g_{ \pm}=2( \pm 3 / 2)$ $+1=4($ for + sign $)$. The values of $g_{+}(n+1 / 2)$ are now,

$$
0,6,10,14,18, \ldots
$$

This series is actually observed in the experimental data. As we can see, there is no need of random topological numbers, no need of Chern numbers and no need of Hofstadter butterfly [10]. The growth of the series such as that in (25) is not a fractal growth and it does not have a constant chemical length.

The $g$ values.

The electron produces its own electromagnetic field which changes the $g$ value. This is a small field but quite noticeable in ordinary electron spin resonance experiments. The magnetic moment of the electron is,

$$
\mu=-\frac{1}{2} g \mu_{B} \frac{S}{\hbar / 2}
$$


where $S$ is the spin. Usually $S=1 / 2$ but in solid state, electron clusters are formed so that it is not limited to $1 / 2$ and it may be 1 , $3 / 2$ or $5 / 2$, etc. The accurate value of $g / 2$ is needed to obtain the magnetic moment of the electron. Therefore, it is important to calculate the energy contributions of the electron-photon interaction which can be used to redefine the $\mathrm{g}$ value. Hence, an expansion has been considered,

$$
\begin{aligned}
\frac{1}{2} g= & 1+c_{2}(\alpha / \pi)+c_{4}(\alpha / \pi)^{2}+c_{6}(\alpha / \pi)^{3} \\
& +c_{8}(\alpha / \pi)^{4}+c_{10}(\alpha / \pi)^{5}+\ldots
\end{aligned}
$$

in which all of the coefficients have been carefully calculated to find,

$$
\alpha^{-1}=137.035999084(33)
$$

These calculations are limited to $l=0, \mathrm{~s}=1 / 2$ only. Therefore, two values of $g$ are not obtained. Even then there are two values due to the \pm in (12). One of these values is zero and the other is 2 besides the electrodynamic correction which is known for $l=0$. Let us take only 2 terms and substitute 0 and 2 for the $g$ value. Then we obtain two equations,

$$
\begin{aligned}
& \frac{1}{2}\left(2+g_{e d}\right)=1+c_{2}^{(+)}\left(\alpha^{(+)} / \pi\right) \\
& \frac{1}{2}\left(0+g_{e d}\right)=1+c_{2}^{(-)}\left(\alpha^{(-)} / \pi\right)
\end{aligned}
$$

leaving out small terms. The solution of the second of these gives negative value for $c_{2}{ }^{(-)} \alpha^{(-)}$, which means that $c_{2}{ }^{(+)}$ is not equal to $c_{2}{ }^{(-)}$. Therefore, the values of the coefficients depend on the $g$ values. The sign of the spin is contained in the $g$ value so that both the positive as well as negative spin values are important.

\section{- Two Constants}

The resistivity at $n=0$ in eq. (24) for positive sign of the spin is,

$$
\rho=\frac{h}{\frac{1}{2} g_{ \pm} e^{2}}
$$

where $g_{ \pm}$must be taken from (12) and it is free from the electrodynamic effects. We list some of the values which give the quantization of the resistivity [10], [11]:
1) $h=6.626068960(330) \times 10^{-34} \mathrm{Js}$
2) $e=1.602176487(40) \times 10^{-19}$ Coulomb
3) $h / e^{2}=25812.8075651 \mathrm{Ohm}$ [pencil calculation]
4) $(1 / 2) \mathrm{g}=1.001159652180$ 73(28) [Hanneke et al. 2008]
5) $h / e^{2}=25812.807557(18)$ Ohm [ CODATA 2006] [11].

By taking only two terms from the right hand side of (27), we find that charge can be completely eliminated,

$$
\rho=\frac{h}{e^{2}}=\frac{h c_{2}{ }^{\prime}}{\pi\left(4 \pi \varepsilon_{o} \hbar c\right)\left(\frac{g}{2}-1\right)}
$$

but the two values of the resistivity are exactly equal to each other. The error in the experimental value of $25812.8 \Omega$ is perhaps not more than $\pm 0.20 \Omega$. The expression (12) gives the doubling of values due to \pm signs and gives the correct fractional values of the charges which agree with the measured values.

- The spin density

There is a special case when $(1 / 2) g=1$,

$$
\frac{1}{2} g_{ \pm}=\frac{l+\frac{1}{2} \pm s}{2 l+1}
$$

which occurs for $l=0, \mathrm{~s}=+1 / 2$. For this case the resistivity (30) is the same as von Klitzing's value. In cases of finite $l$ and s, the physics of the problem is different from that of von Klitzing et al, so that von Klitzing's constant becomes a special case of "spin-dependent" phenomenon [3]. The values of $\rho_{K(+)}$ and $\rho_{K(-)}$ from the expression,

$$
\rho_{K( \pm)}=\frac{h}{\frac{1}{2} g_{ \pm} e^{2}}
$$

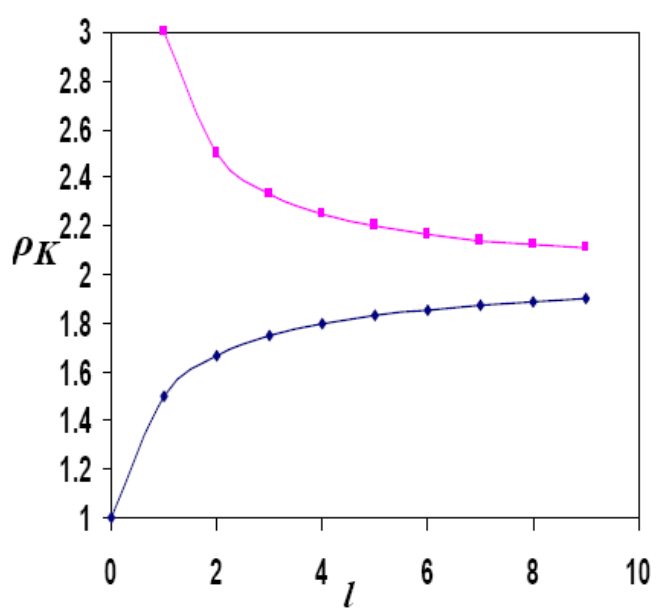

Fig. 1. The variation of resistivity as a function of $l$. The upper curve is (-) spin and lower curve is (+) spin. See text eq.(32)

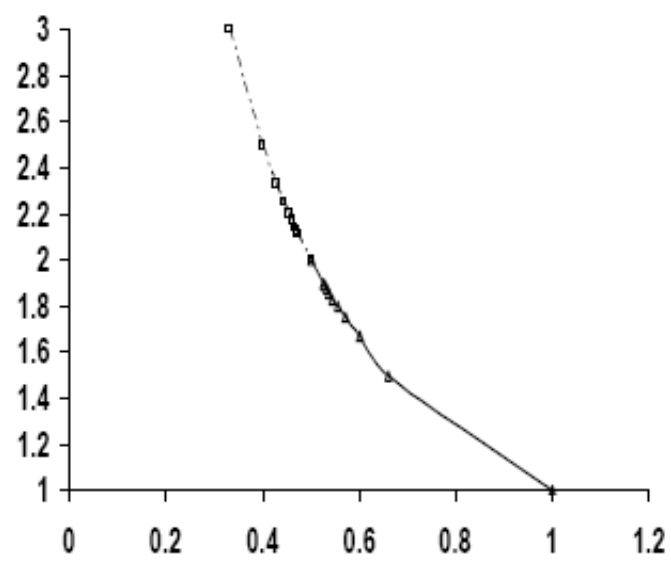

Fig. 2. Plot of resistivity as a function of $(1 / 2) g$. The continuous line on the right hand side of 0.5 has (+) spin and that on the left hand side of 0.5 has (-) spin

are given in Table I along with the values of $g_{ \pm}$. A plot of $\rho_{K( \pm)}$ as a function of $l$ is given in Fig. 1 .

At $l=0, \rho_{\mathrm{K}}=1$, we obtain the von Klitzing's constant. 
Otherwise, there are many values and the von Klitzing constant is a special case of more general constants:

$$
\rho_{K(+)}(l=0, \mathrm{~s}=1 / 2)=\text { Von Klitzing's constant }
$$

$\rho_{K( \pm)}(l \neq 0, \mathrm{~s}= \pm 1 / 2, \pm 1, \pm 3 / 2, \pm, 2, \ldots)=$ General constants

A plot of $\rho_{K( \pm)}$ as a function of $(1 / 2) g$ from Table I is given in Fig. 2.

When $(1 / 2) g=1$, we obtain the von Klitzing's constant, otherwise, more general constants exist.

TABLE I: THE VALUES OF VARIOUS CONSTANTS

\begin{tabular}{|l|l|l|l|l|l|}
\hline S.No. & $l$ & $\rho_{\mathrm{K}(+)}$ & $\rho_{\mathrm{K}(-)}$ & $(1 / 2) \mathrm{g}_{+}$ & $(1 / 2) \mathrm{g}-$ \\
\hline 1 & 0 & 1 & $\infty$ & 1 & 0 \\
\hline 2 & 1 & $3 / 2$ & $3 / 1$ & $2 / 3$ & $1 / 3$ \\
\hline 3 & 2 & $5 / 3$ & $5 / 2$ & $3 / 5$ & $2 / 5$ \\
\hline 4 & 3 & $7 / 4$ & $7 / 3$ & $4 / 7$ & $3 / 7$ \\
\hline 5 & 4 & $9 / 5$ & $9 / 4$ & $5 / 9$ & $4 / 9$ \\
\hline 6 & 5 & $11 / 6$ & $11 / 5$ & $6 / 11$ & $5 / 11$ \\
\hline 7 & 6 & $13 / 7$ & $13 / 6$ & $7 / 13$ & $6 / 13$ \\
\hline 8 & 7 & $15 / 8$ & $15 / 7$ & $8 / 15$ & $7 / 15$ \\
\hline 9 & 8 & $17 / 9$ & $17 / 8$ & $9 / 17$ & $8 / 17$ \\
\hline 10 & 9 & $19 / 10$ & $19 / 9$ & $10 / 19$ & $9 / 19$ \\
\hline 11 & $\infty$ & 2 & 2 & $1 / 2$ & $1 / 2$ \\
\hline
\end{tabular}

\section{TURNING POINTS}

As the gate voltage is increased, the resistivity starts turning towards the plateau. This phenomenon can occur when spins start turning. When the resistivity is at the Hall effect value, away from the plateau region, the electron spin starts turning until the area is so adjusted as to satisfy the flux quantization, which means that the vortex area becomes an integer multiple of flux quanta divided by the field, area = $\mathrm{n} \varphi_{\mathrm{o}} / \mathrm{B}$. The area in the Hall region is infinite. As the spins turn, the area starts reducing from the infinite value to the quantized value.

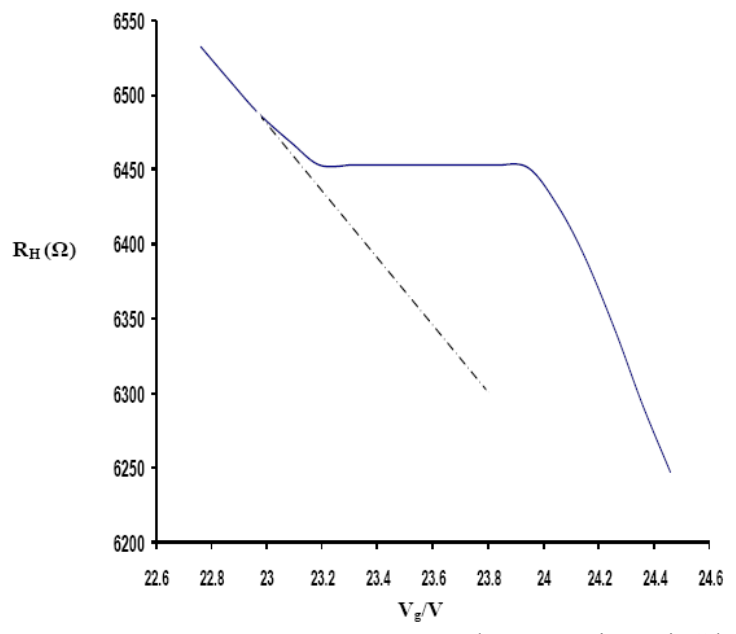

Fig. 3. As the gate voltage is increased, the data shows "turning point" before reaching the plateau.

The change in resistivity from the turning point to the plateau is about $72.7 \mathrm{Ohm}$ compared with $\mathrm{h} / 4 \mathrm{e}^{2}=6453.201$ Ohm.

A plot of the resistivity as a function of gate voltage is given in Fig. 3. At the turning point, the resistivity is,

$$
\rho_{\text {turn }}=6471.21 \Omega
$$

compared with the pencil calculation of $\mathrm{h} / 4 \mathrm{e}^{2}=6453.03 \Omega$. These two values are off by $18.18 \Omega$. In order to compare the turning point value with the plateau value, we define,

$$
\delta \rho=\rho_{\text {turn }}-\rho_{\text {plateau }}
$$

Then the value of $\rho_{\text {plateau }}$ is $25812.8075 \Omega$ whereas $\rho_{\text {turn }}(i=1)$ is $25884.84 \Omega$ so that,

$$
\delta \rho=72.0 \Omega
$$

This in principle makes the values of $h / i e^{2}$ ( $i=$ integer). int"single electron" type theory is sufficient to obtain the value quite uncertain. The experimental uncertainty in the value of 25812.8 is only $0.2 \Omega$ but then in principle uncertainty is $2.8 \times 10-3$ which is a few parts per thousand. The plateau measurement is obviously much more curate than the difference between turning point and the plateau. In such a case, in principle value will play a dominant role. The plateau value can be measured upto eight digits which means that the accuracy is 1 part in 108 , If that is the case the plateau is sharply peaked but the distribution may be extended upto the turning point. It is said that the center of a line can be located to a large accuracy. That does not mean that there is no line width. The line is an envepole of a large number of events so that there is a finite width. The accuracy of measurements is thus not the accuracy of locating the plateau but the location of the turning point. In Laughlin's work an effort is made to obtain this error factor by the calculation of correlations. A single electron hype theory is sufficient to obtain the values at the plateaus.

A "single electron" type theory is sufficient to obtain the value of e and there are no many-body effects which can change the value of $e$ [4]. The experimentally measured error is not more than $\pm 0.2 \Omega$ in $25812.8 \Omega$, which means that we can get the values correct upto ppm [2]. This point is to be noted so that we are not carried away from the "single particle theory" [3]-[6] of the observed plateau in the Hall resistivity. Another observation is that plateaus are formed and destroyed as the magnetic field is varied. If there is considerable variation in the sample size and temperature, more than 101 plateaus can be observed [7]. To reduce the problem to a tractable size, we can consider only two plateaus and the interventing phase so that as the field is varied, there are three phases. The electrons in the intermediate phase are subject to the many-body interactions such as Coulomb interactions, electron-phonon interactions, interacting Landau levels, etc. Hence the problem is to understand the phase transition from a many-body interacting phase to a single-particle non-interacting phase. The flux quantization fixes the area at $A=\left(n^{\prime} h \mathrm{c}\right) /\left(e^{*} B\right)$ so that we know the area occupied by the electrons in the plateau region which is defined by the resistivity and the magnetic field, $\rho_{x y}=h / i e^{2}$, where I can be an integer. It is also possible that I is a fraction which depends on angular momentum. The area in between plateaus need not be the same as in the flux quantization. Hence, the area can also be used to demark the phase boundaries. The electrons move from the conduction band to 
a localized band which is flux quantized. Hence, the flux-quantized plateau which belongs to a single-particle state is also Anderson localized except that the Anderson localization has to be modified for spin.

We report our study of the phase transitions which occur as the resistivity goes from one plateau to another. The intermediate phase in between two plateaus, is also important because it is associated with a clustering phase.

\section{The PHASES AS A FUNCTION OF FIELD}

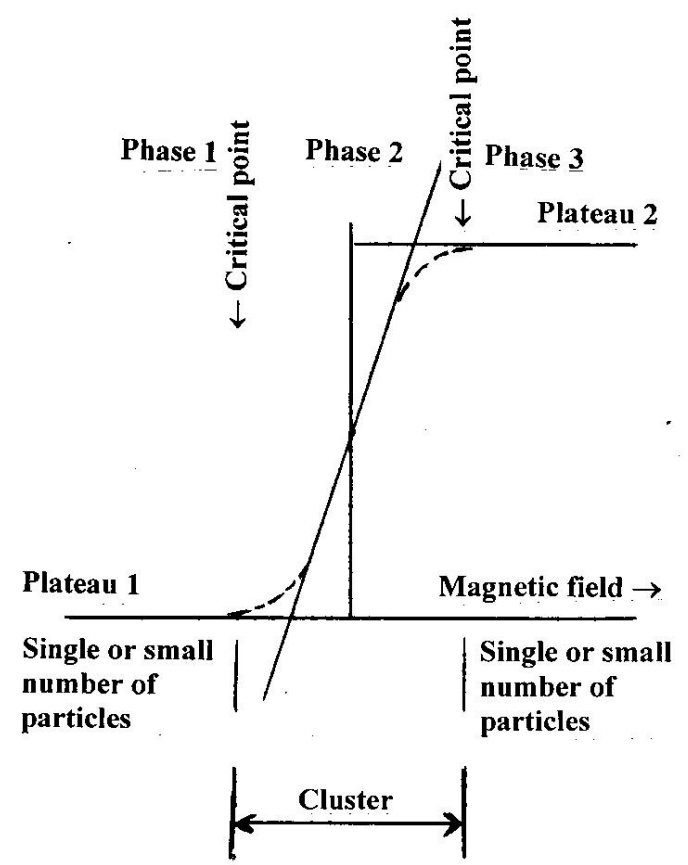

Fig. 4. Two plateaus and the intervening cluster phase. The plateaus have a single particle or small number of particle state whereas the cluster has a lot of particles.

We consider a phase diagram which has three phases as shown in Fig. 4. The phase-1 is characterized by a plateau at $v_{1}$. The resistivity in this phase is $h /\left(v_{1} e^{2}\right)$. Since $v_{1}$ is exactly defined, it corresponds to a single electron with well defined $l$ and $s$ values. For a single electron the spin is $s=1 / 2$ and it has a well defined value of $l$ which gives the orbital angular momentum $l \hbar$. In some cases, the single electron is replaced by a few electrons so that spin need not be $1 / 2$ but it can be a different value such as 1 or $3 / 2$, etc. Since, the charge is fixed at $e^{*}=v_{1} e$, it corresponds to a fixed area in which flux is quantized. Hence the area is, $n^{\prime} h c / e^{*} B$. The value of $v_{1}$ is fixed by [12]-[19],

$$
\frac{e^{*}}{e}=v_{1}=\frac{1}{2} g_{ \pm}=\frac{l_{1}+\frac{1}{2} \pm s_{1}}{2 l_{1}+1}
$$

In the phase-1, the orbital angular momentum is $l_{1}$ and the spin of a particle is $s_{1}$. The value of $v_{1}$ can be zero for $l_{1}=0$, $s_{1}=1 / 2$ with negative sign. It can be $v_{1}=1$ for positive sign and $s_{1}=1 / 2, l_{1}=0$, etc. Some other values of $v_{1}$ are also possible. There is a critical point at which the plateau ends, more electrons come in the area which expands to form the phase- 2 which is a cluster with area not determined by flux quantization but it corresponds to a critical exponent. Since, the area is expanding, it may vary as the square of the coherence length,

$$
\xi=\xi_{0}\left(B-B^{*}\right)^{-v}
$$

where $B^{*}$ is a constant field and $v$ is the critical exponent. After some increase in the field, the variation of the resistivity as a function of magnetic field becomes constant, $\partial \rho_{x y} / \partial B=$ constant so that its slope can be determined. In this region,

$$
\rho_{x y}=c_{1} B+c_{2}
$$

and we call it "cluster phase". In this phase, the electrons form a cluster. As the field is further increased, the phase-2 which ithe same as the cluster phase, starts breaking down. This starting point is a critical point and then with further increase in the field another plateau is built. This another plateau is the phase- 3 and it has its own $l$ and $s_{2}$. It is found that in going from phase- 1 to phase- 3 , if 1 increases, then it is accompanied with a decrease of $\mathrm{s}$ which can be done by choosing the negative sign in s.

\section{The Phases as a Function of Temperature}

The $\partial \rho_{x y} / \partial B$ clearly has two values, one below $10 \mathrm{mK}$ and the other from $0.01 \mathrm{~K}$ to $1.0 \mathrm{~K}$ [20]. From eq.(40) we see that $\partial \rho_{x y} / \partial B=c_{1}$. This constant $\mathrm{c}_{1}$ is independent of the magnetic field but it varies with temperature, $c_{1}=c_{1}(\mathrm{~T})$. In the temperature range $0.01-1.0 \mathrm{~K}$, the shift from phase- 1 to phase- 3 is very smooth at high temperatures of the order of $500 \mathrm{mK}$ and very steep at low temperatures such as $15 \mathrm{mK}$. Hence, it is possible to define a critical exponent in the "cluster phase" as,

$$
\partial \rho_{x y} / \partial B \sim T^{\kappa}
$$

Such an exponent should occur symmetrically in going from phase-1 to phase-2 and in going from phase-2 to phase-3. Since, it is a problem of coherence length, it is possible that $\kappa=1 / \nu$ where $v$ is the exponent of the divergence of the coherence length,

$$
\xi=\xi_{o}\left(1-\frac{T}{T_{c}}\right)^{-v}
$$

The experimental data has two values [8], one below 10 $\mathrm{mK}$ and the other between 0.01 to $1.0 \mathrm{~K}$. Apparently, $\kappa=1 / \nu=0.42$ is consistent with the coherence length effect. A more interesting problem is found below $10 \mathrm{mK}$. These experiments are also more difficult to do because of noise induced heating and hence bigger error bars than at higher temperatures. In any case, this is a good opportunity to measure the radiative effects. At this temperature, the phonons are completely eliminated except the zero-point vibration so that we expect to see the effect of self electromagnetic fields of the electron. The conductivity is proportional to the radiative life time of the electron. At this temperature, there is a finite life time, usually quite long, so that there is a small radiative contribution to the resistivity. In 
the clustering region, this effect may be important.

\section{LANDAU LEVELS}

The electrons in two dimensions behave like harmonic oscillators with eigen values given by $\hbar \omega_{c}(n+1 / 2)$. Here $\omega_{c}$ is the cyclotron frequency and $\mathrm{n}$ is an integer. For $\mathrm{n}=0,(1 / 2) \hbar \omega_{c}$ is the lowest Landau level (LLL). The effective charge of a quasiparticle is then,

$$
\frac{e_{e f f}}{e}=\frac{1}{2} g_{ \pm}\left(n+\frac{1}{2}\right)
$$

In $g_{ \pm}$we have to change the orbital magnetic moment by changing $l$ and s. The Landau levels are derived for only one plateau at a time so that we can tabulate the various effective charges. But we cannot go from one Landau level to another.

The size of the vortex from the Compton wave length is $3.9113 \times 10^{-15} \mathrm{~cm}$. We assume that one vortex is attached to the electron such that only the reduced mass appears in the Schroedinger equation. Making the vortex electron pair has no effect on the Hall effect.

\section{CONCLUSIONS}

The positions of the plateaus are correctly predicted [13]-[20] by the angular momentum theory [1]. The region in between plateaus show the formation of clusters of electrons. The number of electrons at the plateaus is one or a small number. One can go from the plateau to the cluster region by scaling as in a phase transition. In that case, the plateau region is Anderson localized except that the spin has to be treated as in ref.[15]. There are phase transitions type characteristics in between plateaus.

\section{REFERENCES}

[1] K. N. Shrivastava, "The quantum Hall effecr: Interpretation of the experimental data," International Journal of Applied Physics and Mathematics, vol. 2, no. 6, pp. 467-473, November 2012.

[2] K. N. Shrivastava, "The quantum Hall effecr: Helicity, graphite and grapheme," International Journal of Applied Physics and Mathematics, vol. 3, no. 1, pp. 1467-22473, January 2013.

[3] K. N. Shrivastava, "Universal introduction to the quantum Hall effecr," International Journal of Applied Physics and Mathematics, vol. 3, no. 3, pp. 208-216, May 2013.

[4] K. von Klitzing, G. Dorda, and M. Pepper, "New method for high-accuracy determination of the fine structure constant," Phys. Rev. Lett., vol. 45, pp. 494-497, 11 August 1980.

[5] D. C. Tsui, H. L. Stormer, and A. C. Gossard, "Two-dimensional magnetotransport in the extreme quantum limit," Phys. Rev. Lett., vol. 48 , pp. $1559-1562,1982$
[6] F. D. M. Haldane, "Fractional quantization of the Hall effect: A hierarchy of incompressible quantum fluid states," Phys. Rev. Lett., vol. 51, pp. 605-608, August 1983.

[7] K. N. Shrivastava, "The quantum Hall effect:The symmetry and the velocity, (IOP)," J.Phys:Conf. Ser., vol. 423, 2013.

[8] H. L. Stormer, "Nobel lecture: The fractional quantum Hall effect," Reviews of the Modern Physics, vol. 71, pp. 875-889, July 1999.

[9] A. Luican, Q. Li, A. Raina, J. Kong, R. R. Nair, K. S. Novoselov, A. K. Geim, and E. Y. Andrei, "Single layer behavior and its breakdown in twisted graphene layers," Physical. Review. Letters, vol. 106, no. 12 , March 2012

[10] D. A. Abanin, K. S. Novoselov, U. Zeiller, P. A. Lee, A. K. Geim, and L. S. Levetov, "Dissipative quantum Hall effect in graphene near the Dirac point," Physical Review Letters, vol. 98, May 2007.

[11] P. J. Mohr, B. N. Taylor, and D. B. Newell, Phys. Today 60, vol. 52, 2007.

[12] R. B. Laughlin, Phys. Rev. B, vol. 23, pp. 5632, 1981.

[13] R. B. Laughlin, Phys. Rev. Lett., vol. 50, pp. 1395, 1983.

[14] A. N. Rosli and K. N. Shrivastava, in Proc. AIP Conf., vol. 1136, pp. 469-473, 2008.

[15] K. N. Shrivastava, Phys. Lett. A, vol. 113, pp. 435, 1986.

[16] K. N.Shrivastava, Mod. Phys. Lett., vol. 13, pp. 1087, 1999.

[17] K. N. Shrivastava, Mod. Phys. Lett., vol. 14, pp. 1099, 2000.

[18] K. N. Shrivastava, Phys. Lett. A, vol. 326, pp. 469, 2004.

[19] K. N. Shrivastava, in Proc. AIP Conf., vol. 1150, pp. 59-67, 2009.

[20] W. Li, C. L. Vicente, J. S. Xia, W. Pan, D. C. Tsui, L. N. Pfeiffer, and K. W. West, Phys. Rev. Lett., vol. 102, pp. 216801, 2009.

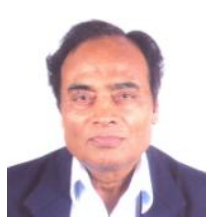

Keshav N. Shrivastava was born on July 11, 1943. He obtained B.Sc. degree from the Agra University in 1961, M.Sc. degree from the University of Allahabad in 1963, Ph.D. from the Indian Institute of Technology Kanpur in 1966 and D.Sc. from Calcutta University in 1980.

He worked at the Clark Universityin USA during 1966-68, at the Harvard University USA during the summer of 1968, at the Montana State University USA during 1968-69, and at the University of Nottingham during 1969-70. He was Associate Professor at the Himachal Pradesh University Simla, India during 1970-74. He worked at the University of California Santa Barbara USA during 1974-75 and at the State University Utrecht, the Netherlands, during 1975-76. He was Professor at the University of Hyderabad where he worked from 1978 till $2005 . \mathrm{He}$ worked in Tohoku University Japan during 1999-2000. He visited the University of Zurich Switzerland for short times during 1984, 1985, 1988 and 1999. He worked in the University of Houston USA in the summer of 1990. He visited the Royal Institute of Technology Stockholm Sweden and the University of Uppsala Sweden during 1994. He visited the Technical University of Vienna, Austria in the summer of 1995. He visited the Princeton University USA and the University of Cincinnati USA during 2003. He was Professor in the University of Malaya Kuala Lumpur Malaysia during 2005-2011. He is affiliated with the University of Hyderabad, India. He has worked in electron spin resonance, spin-phonon interaction, relaxation, Moessbauer effect, exchange interaction, superconductivity and the quantum Hall effect. He discovered the flux quantized energy levels in superconductors, the spin dependent flux and the correct theory of the quantum Hall effect. He has published more than 232 papers in journals and he is the author of the following books. 1) Superconductivity: Elementary Topics, World Scientific Singapore 2000. 2) Introduction to Quantum Hall effect, Nova Sci. Pub N.Y. 2002 and 3) The quantum Hall effect: Expressions, Nova Sci. N.Y. 2005.

Prof. Shrivastava is a fellow of the Institute of Physics London, Fellow of the National Academy of Sciences India and member of the American Physical Society. 